\title{
Low-Molecular-Weight Heparin (LMWH) in Women with Repeated Implantation Failure
}

\author{
Allahbadia Gautam N.
}

Published online: 13 October 2012

(C) Federation of Obstetric \& Gynecological Societies of India 2012

\section{Introduction}

Despite the initial dramatic improvements in success rates and significant increments in uptake of assisted reproduction techniques (ARTs), the live birth rate resulting from these techniques has recently plateaued [1]. Greater emphasis is now being placed on experimental strategies to further improve oocyte and embryo number and quality, implantation rates, and successful transition to live births. One such strategy is the use of low-molecular-weight heparin (LMWH) which, as a product having significant impact on live birth rates in women with acquired thrombophilia [2,3], is now being considered as a potential therapy for all ART patients. Although the effect of LMWH on trophoblast biology has not been studied extensively, given the available data suggesting a possible beneficial effect of LMWH on embryo implantation, we use LMWH extensively in ART cycles especially in the group of patients with recurrent implantation failures RIF with consistently uncompromised embryo quality. These benefits will include reductions in the thrombotic risks associated with exogenous gonadotropins; and, given the accumulating evidence that the beneficial effects of LMWH are not solely mediated via an anticoagulant effect [4], the potential for LMWH being used for optimizing implantation and trophoblast development.

Allahbadia G. N. ( $₫)$, Medical Director

Rotunda-The Center for Human Reproduction, 36 Turner Road, \#101, 1st Floor, B Wing, Bandra (W), Mumbai 400 050, India e-mail: gautam@rotundaivf.com

\section{Discussion}

Recently, thrombophilia (acquired and inherited) has been implicated in recurrent IVF-ET failure. The objective of the study by Qublan et al. [5] was to determine the effect and safety of thromboprophylaxis using LMWH in women with recurrent in vitro fertilization (IVF)-embryo transfer (ET) failure and thrombophilia. Eighty-three women with history of three or more previous IVF failures and who had at least one thrombophilic defect were eligible for this study. Patients were randomly allocated into two groups: Group A $(n=42)$ that received enoxaparin $40 \mathrm{mg} /$ day, and group B $(n=41)$ that received placebo $(\mathrm{NaCl} 0.9 \%)$. Both treatments started on the day of ET and continued until delivery or fetal demise was diagnosed. The primary outcomes were the implantation, pregnancy, and live birth rates. Patients who received LMWH for thromboprophylaxis had a significant increase in the implantation and pregnancy rates compared with the placebo group (20.9 vs. $6.1 \%$ and 31 vs. $9.6 \%$, respectively; $p<0.001$ and $p<0.05$, respectively). A significant increase in the live birth rate was observed in the LMWH-treated group compared with placebo-treated group ( 23.8 vs. 2.8 , respectively; $p<0.05$ ). The abortion rate was significantly higher in the placebo-treated group compared with the heparin-treated group $(p<0.05)$.

Noci et al. [6] evaluated whether heparin administration could affect IVF outcome. A total of 172 women, aged $<40$ years, without laboratory findings of thrombophilia and undergoing their first IVF cycle, were randomly allocated to treatment $(n=86)$ and control $(n=86)$ groups. Patients 
allocated to the treatment group received LMWH dalteparin sodium 2,500 IU s.c. daily, in addition to routine luteal phase support, from oocyte retrieval up to the day of the pregnancy test or up to the ninth week of pregnancy in the cases of positive human chorionic gonadotropin. Implantation rates were 15 and $12 \%$ in the dalteparin and control groups, respectively. The clinical pregnancy rates/embryo transfers were 26 (19/73) and $20 \%$ (16/80), in the dalteparin and control groups, respectively, with live birth rates/embryo transfer of $21(15 / 73)$ and $16 \%$ (13/80). Despite the lack of statistical significance, the increase in pregnancies observed in the treatment group may be considered as an important clinical point in the optimization of IVF clinical outcome [6].

One hundred and fifty women with $>$ or $=2$ failed assisted reproduction treatment cycles were included in a randomized open-label pilot trial by Urman et al. [7]. Participants underwent controlled ovarian stimulation with the long protocol and were randomly allocated to receive $1 \mathrm{mg} / \mathrm{kg} /$ day LMWH or no treatment in addition to routine luteal phase support (LPS) on the day after oocyte retrieval. LPS and LMWH were continued up to the 12th gestational week in pregnant participants. There were $26(34.7 \%)$ live births in the LMWH group, and $20(26.7 \%)$ in the control group (absolute difference 8.0, $95 \% \mathrm{CI}-4.2$ to $24.9 \%$, $p=0.29$ ). They concluded that despite lack of statistical significance, observed relative increase by $30 \%$ in live birth rates with LMWH may be regarded as a clinically significant trend necessitating further research on the use of empirical LMWH in women with RIF and possibly in all women undergoing assisted reproduction treatment [7].

Jersak et al. [8] reported a strikingly remarkable case with 15 IVF failures. When enoxaparin was added, the 16th IVF cycle generated a healthy male baby. Those authors concluded that combination therapy that includes a LMWH might allow successful IVF outcome.

Different mechanisms of action besides anticoagulation can be suggested for the beneficial effects of heparin on implantation [9]. These include the following:

E-cadherin is an intercellular adhesion molecule that has been shown to be involved in trophoblast differentiation and invasiveness [10]. LMWH may facilitate implantation process by down-regulating the E-cadherin expression in the decidua.

Tightly regulated expression of HB-EGF in luminal epithelium and of EGF-receptor in the blastocyst around the time of implantation suggests an important function for this ligand-receptor signaling in embryo-maternal crosstalk for implantation [11]. Heparin, via heparan sulfate proteoglycans or HB-EGF, may be involved in blastocyst adhesion and invasion [2, 9]. Furthermore, HB-EGF was shown to enhance in vitro extravillous trophoblast differentiation [12].

Enhanced trophoblast migration and invasiveness due to a LMWH-induced increase in free insulin-like growth factor I is another proposed mechanism for a beneficial effect of LMWH on the implantation process [9].

Antibodies against annexin- $\mathrm{V}$, a potent anticoagulant abundant in placental tissues, were recently controversially reported to be associated with recurrent miscarriages or failures of in vitro fertilization (IVF) attempts. Halbmayer et al. [13] screened 56 women $(34.7 \pm 4.3$ years of age: mean \pm 1 SD) with recurrent IVF failures and/or early pregnancy losses for resistance against activated protein $\mathrm{C}$, lupus anticoagulant, and antibodies against annexin $\mathrm{V}$, cardiolipin, or beta(2)-glycoprotein-1. Among them, the prevalence of APC-R (8/56, $14 \%)$ and elevated levels of IgG- or IgM-anti-cardiolipin antibodies $(7 / 56,12 \%)$ were more common than elevated levels of (IgG or IgM) antibodies against beta(2)-glycoprotein-1 (3/56, $5 \%$ ) or annexin-V (1/56, $2 \%) .42(75 \%)$ of the women had another IVF-attempt after this hemostaseological evaluation and received LMWH and/or acetylsalicylic acid in the case of positivity for APC-resistance, lupus anticoagulant, or antibodies against annexin V, cardiolipin, or beta(2)-glycoprotein1. The outcome of these IVF-attempts was 19 pregnancies (34\%): four early miscarriages (7\%) and 15 so far uncomplicated pregnancies $(27 \%)$. The only woman with an elevated anti-annexin $\mathrm{V}(\mathrm{IgG})$ level had 7 IVFs before and received $40 \mathrm{mg}$ enoxaparin s.c. once daily during the 8th IVF, which resulted in a healthy pregnancy. The findings suggest that among women with recurrent IVF failures, anti-annexin $\mathrm{V}$ antibody positivity is less prevalent than APC-resistance, lupus anticoagulant (LA), or elevated levels of antibodies against cardiolipin, beta (2)-glycoprotein-1; and that the IVF-result of women with APC-R, LA, or with elevated levels of antibodies against annexin $\mathrm{V}$, cardiolipin or beta(2)-glycoprotein might be positively influenced by low molecular weight heparin [13].

Lodigiani et al. [14] performed a retrospective observational analysis of patients with at least two IVF/intracytoplasmic sperm injection cycles with implantation failure, screened for inherited thrombophilia and submitted to further ART cycles with or without administration of LMWH. A total of 265 patients fulfilled the enrollment criteria. They analyzed basal FSH, smoking habit, gene variants for inherited thrombophilia (i.e., MTHFR C677T, prothrombin G202A10G, and Factor V Leiden). The patients underwent 569 new ART cycles: 512 (90\%) without and $57(10 \%)$ with LMWH. Significantly higher pregnancy rate in patients with previous ART implantation failures was observed with LMWH [14].

On the other hand, Bohlmann performed a systematic literature search of the PubMed/Medline database assessing all the articles published before November 2010 [15]. He summarized that the available studies on LMWH in assisted reproduction are characterized by heterogeneous inclusion criteria and a lack of proven effectiveness in special constellations. He concluded that the application of LMWH to improve ART outcome rates is not justified [15]. 


\section{Conclusions}

Implantation failure is common in ART. The role of LMWH as a potential factor to improve implantation is a subject matter of debate. The pathology underlying RIF is not clear, and treatment options proposed are generally not evidence based. Heparin can alter the hemostatic response to the controlled ovarian stimulation and modify the risk of thrombosis. It can also modulate many of the fundamental physiological processes required for blastocyst apposition, adherence, and implantation, as well as trophoblast differentiation and invasion due to its similarities with heparan sulfates and has the potential to improve pregnancy rates and outcomes.

Despite in vivo and in vitro evidences in favor of the alleged beneficial effect [2, 5-7, 9], wholesale adoption of this strategy into routine practice will not become a gold standard until further research confirms the presence of such an effect and reveals its exact mechanism of action.

\section{References}

1. Allahbadia GN, Kadam K, Gandhi G, et al. Embryo transfer using the SureView catheter-beacon in the womb. Fertil Steril. 2010;93(2):344-50.

2. Fiedler K, Wurfel W. Effectivity of heparin in assisted reproduction. Eur J Med Res. 2004;9:207-14.

3. Empson M, Lassere M, Craig J, et al. Prevention of recurrent miscarriage for women with antiphospholipid antibody or lupus anticoagulant. Cochrane Database Syst Rev. 2005;2:CD002859.

4. Girardi G, Redecha P, Salmon JE. Heparin prevents antiphospholipid antibody-induced fetal loss by inhibiting complement activation. Nat Med. 2004;10:1222-6.
5. Qublan H, Amarin Z, Dabbas M, et al. Low-molecular-weight heparin in the treatment of recurrent IVF-ET failure and thrombophilia: a prospective randomized placebo-controlled trial. Hum Fertil (Camb). 2008;11(4):246-53.

6. Noci I, Milanini MN, Ruggiero M, et al. Effect of dalteparin sodium administration on IVF outcome in non-thrombophilic young women: a pilot study. Reprod Biomed Online. 2011;22(6):615-20.

7. Urman B, Ata B, Yakin K, et al. Luteal phase empirical low molecular weight heparin administration in patients with failed ICSI embryo transfer cycles: a randomized open-labeled pilot trial. Hum Reprod. 2009;24(7):1640-7.

8. Jerzak M, Niemiec T, Nowakowska A, et al. First successful pregnancy after addition of enoxaparin to sildenafil and etanercept immunotherapy in woman with fifteen failed IVF cyclescase report. Am J Reprod Immunol. 2010;64(2):93-6.

9. Nelson SM, Greer IA. The potential role of heparin in assisted conception. Hum Reprod Update. 2008;14(6):623-45.

10. Xue WC, Feng HC, Tsao SW, et al. Methylation status and expression of E-cadherin and cadherin-11 in gestational trophoblastic diseases. Int J Gynecol Cancer. 2003;13:879-88.

11. Hamatani T, Daikoku T, Wang H, et al. Global gene expression analysis identifies molecular pathways distinguishing blastocyst dormancy and activation. Proc Natl Acad Sci USA. 2004;101:10326-31.

12. Leach RE, Kilburn B, Wang J, et al. Heparin-binding EGF-like growth factor regulates human extravillous cytotrophoblast development during conversion to the invasive phenotype. Dev Biol. 2004;266:223-37.

13. Halbmayer WM, Feichtinger W, Kindermann C, et al. Recurrent miscarriage or failed in vitro fertilization: antibodies against annexin V, cardiolipin, beta-2-glycoprotein-1 and APC-resistance. Hamostaseologie. 2005;25(4):391-3.

14. Lodigiani C, Di Micco P, Ferrazzi P, et al. Low-molecular-weight heparin in women with repeated implantation failure. Womens Health (Lond Engl). 2011;7(4):425-31.

15. Bohlmann MK. Effects and effectiveness of heparin in assisted reproduction. J Reprod Immunol. 2011;90(1):82-90. 